\title{
Editorial \\ Rich models in discrete optimization: Formulation and resolution (ECCO XVI)
}

This special issue of European Journal of Operational Research is devoted to the ECCO XVI Conference, the sixteenth annual meeting of the European Chapter on Combinatorial Optimization, held at the Molde University College (Norway), from June 5 to June 7, 2003. (You can still visit the conference web site at http://www.himolde.no/arrang/eccoXVI/.)

ECCO, the EURO Working Group on Combinatorial Optimization, was created in 1987 by C. Roucairol, D. De Werra and A. Rinnooy Kan. It provides an excellent opportunity to discuss recent and important issues in combinatorial optimization and its applications. The group, which has about 700 members, is open to everybody interested in the field, either in theoretical aspects or in business, industry or public administration applications. During the first ten years, Catherine Roucairol (Université de Versailles, France) coordinated the ECCO activities. The current coordinator is Silvano Martello (University of Bologna, Italy).

The ECCO meetings are held on a regular basis (once a year during Spring) and are devoted to all aspects of combinatorial optimization: operations management and logistics, production scheduling, location and distribution problems, resource allocation, flexible manufacturing, scheduling, VLSI design and computer design, network optimization, etc. At ECCO meetings usually about 100 participants nicely combine scientific works and exchange of new ideas with an exciting atmosphere like Paris (May 1988), Venice (June 1989), Barcelona (May 1990), Dubrovnik (May 1991, canceled due to the unstable political situation in Croatia), Graz (April 1992), Bruxelles (April 1993), Milano (February 1994), Poznan (May 1995), Dublin (April 1996), Tenerife (May 1997), Copenhagen (May 1998), Bendor (May 1999), Capri (May 2000), Bonn (2001), Lugano (2002), Molde (2003), Beirut (2004), Minsk (2005).

The Scientific Program Committee for ECCO XVI was formed by Jacek Błażewicz (Technical University of Poznan, Poland), Van-Dat Cung (University of Versailles, France; now at GILCO Laboratory, Grenoble), Alain Hertz (Université de Montréal, Canada), Arne Løkketangen (Molde Univer- 
sity College, Norway), Silvano Martello (University of Bologna, Italy), and Paolo Toth (University of Bologna, Italy). The meeting was organized by Arne Løkketangen. We would like to thank Oyvind Halskau , Grete Notøy, Berit Svendsvik and Stein W. Wallace, all from the Molde University College, for their service in the Organizing Committee. The conference dinner was held at the Finnøya sjøhus, a restaurant with atmosphere located on a charming island (http://www.havstuene.no/), that was reached with a high speed passenger express boat.

During the conference, the following plenary talks were given:

- Piotr Formanowicz (Institute of Computing Science, Poznan University of Technology and Polish Academy of Sciences), "On some combinatorial problems of computational biology";

- Claude Le Pape, ILOG S.A., "Two generic schemes for efficient and robust cooperative algorithms";

- Stein W. Wallace, Molde University College. "The role of stochastics in optimization: Why it is dangerous to overlook it".

Additionally, sixteen sessions were organized, in two or three parallel streams, on the following main topics: vehicle routing, scheduling, metaheuristics, networks and combinatorics. In these sections, 39 papers were presented and discussed. After the conference nineteen papers have been submitted to the special issue "Rich models in discrete optimization: Formulation and resolution" and, after a thorough refereeing process, nine papers have been selected for publication. We would like to thank Mrs. Rita Mambelli, secretary at the University of Bologna, for her help in organizing and handling the refereeing process.

The objective of this special issue is to investigate the formulation of rich models in discrete optimization, and the development of accompanying exact and heuristic solution methods, in all kinds of real-world application. As computers are getting progressively faster and solution methods are improving, more attempts are made of formulating and solving richer models of complex real-world discrete optimization problems. By rich model, we understand a non-idealized model that represents the application at hand in an adequate way by including all important optimization criteria, constraints, and preferences.

The organizers of ECCO XVI thank all the institutions which supported the meeting. Thanks are due in particular to: 
- City of Molde;

- EURO, the Association of European Operational Research Societies;

- Molde University College.

We also thank SINTEF Applied Mathematics, Møre Research, ILOG and the Norwegian Research Council for their sponsorship.

All the papers of this special issue were strictly refereed. We would like to thank the referees for their fruitful remarks and comments.

Geir Hasle

SINTEF Applied Mathematics, Department of Optimization

Oslo, Norway.

E-mail: Geir.Hasle@sintef.no

http://www.math.sintef.no/opti/staff/gha.html

Arne Løkketangen

Molde University College

Molde, Norway.

E-mail: Arne.Lokketangen@hiMolde.no

http://www.himolde.no/ arnel/

Silvano Martello

DEIS, Università di Bologna

Bologna, Italy.

E-mail: smartello@deis.unibo.it

http://www.or.deis. unibo.it/martello.html 